\title{
Synthesis and Charactrization of New 3, 5 Disubstituted Pyrazoline
}

\author{
N. R. THAKARE \\ Department of Chemistry, Shri Shivaji Science College,Amravati (MS), India. \\ Corresponding author: tnilesh@gmail.com
}

\begin{abstract}
A series of $\mathrm{N}$-(4-chlorophenyl)-3-(4-substitutedphenyl)-acryl amide (3a-c) and $\mathrm{N}$-(4chlorophenyl)-3-furan acryl amide(3d) were synthesized by the simple condensation of 4chloroacetanilide(1) with aromatic aldehydes $(2 a-c)$ and (2d) in presence of $20 \% \mathrm{NaOH}$ as a catalyst. These synthesized acryl amides then treated with phenyl hydrazine and 2,4dinitrophenyl hydrazine in the alcoholic medium to give 3,5-disubstituted pyrazolines (4a-f) and (5d-6d) in a good to excellent yields using a facile approach.

These newly synthesized 3, 5-disubstituted pyrazolines have been characterized on the basis of spectral studies.
\end{abstract}

Keywords :Pyrazolines, 4-Chloroacetanilide, Acrylamide, 3, 5-Disubstituted Pyrazolines

\section{Introduction}

Pyrazolines are well known and important nitrogen contaning 5-membered heterocyclic compounds and various methods have been worked out for their synthesis. Numerous pyrazoline derivatives have been found to posses considerable biological activities which stimulated the research activity in this field. These are antidepressant ${ }^{1,2}$, anticonvulsant ${ }^{3}$, antifungal ${ }^{3}$, antimicrobial ${ }^{4}$,

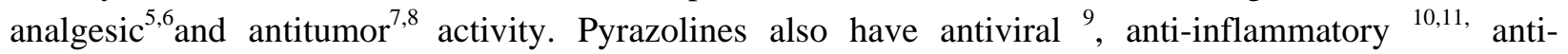
tuberculosis ${ }^{12}$, and anti-amoebic ${ }^{13}$ activity. These compounds are usually prepared from cyclization of chalcones with hydrazine and its derivatives under alcoholic conditions. The major inventive behind the synthesis of these compounds was the immense biological activities associated to these heterocyclic derivatives

\section{Materials and Methods}

4-chloroacetanilide, hydroxylamine hydrochloride, hydrazine hydrate,4-chlorobenzaldehyde,4hydroxy benzaldehyde,ethanol, sodiumhydroxide, vaniline, ferfuraldehyde. Melting points of all synthesized compounds were determine in open capillaries and are uncorrected. The proton NMR spectra were recorded in Bruker AC-II 400 NMR spectrometer in CDCl3 using TMS as a standered. IR spectra were recorded in $\mathrm{KBr}$ pallets using IR spectrometer. The chemicals used were of laboratory reagent grade and the purity of the compounds was checked by TLC

\section{Synthesis of N-(4-Chlorophenyl)-3-Substituted Acryl Amide (3a-d)}

The mixture of 4-chloroacetanilide(1) (0.01 mole) and different aromatic aldehydes like vaniline, p-chloro benzaldehyde, p-hydroxyl benzaldehyde, ferfuraldehyde (0.01 mole) in ethanol $(20 \mathrm{~mL})$ added drop wise to $2.5 \mathrm{~mL} \mathrm{NaOH}(20 \%)$ with stirring. It was then decomposed with ice cold water to obtain the crude product. It was then washed with water, filtered, dried and crystallized from ethanol to get (3a-d) 


\section{Characteristics}

NMR (ㅁ) : 3.87-3.82(S,3H,Ar-OCH3),7.35-3.33(S,1H,OH),9.99-9.95(S,1H,-NH),7.91 -7.74(M,7H,Ar-H) $\operatorname{IR}\left(\mathbf{V}_{\max }\right) \mathrm{cm}^{-1}$ :-3352(O-H),3085(N-H),2857(C-H aliphatic),1697(C=O),1587(C=C),1289(C-N),1204(CO),833(p-sub benzene),682(C-Cl).

Physical data of synthesized compounds (3a-d) given in table no. 1

\section{Synthesis of 3,5-Disubstituted Pyrazolines (4a-f)}

A mixture of $\mathrm{N}$-(4-chlorophenyl)-3-(4-substituted phenyl) acryl amide (0.01 moles) and phenyl hydrazine/2,4 dinitrophenyl hydrazine (0.01 mole) in ethanol was refluxed for 5-6 hours. The resulting mixture was concentrated and allowed to cool. The resulting solid was filtered, washed, dried and recrystallized from ethanol to get (4a-f).

\section{Characteristics}

$\operatorname{NMR}(\underline{\boldsymbol{\delta}})$ :-7.45(s,1H,-OH), 3.5(s,1H,N-H), 7.51-7.45(m,12H,Ar-H), 5.5(d,2H,-CH), 7.1(dd,1H,-CH).

IR $\left(\mathbf{V}_{\text {max }}\right) \mathrm{cm}^{-1}$ :- 3304(O-H),3024(N-H),1588(C=N),1485(C=C),1262(C-O),1203(C-N),816(P-sub.benzine ring),625(C-Cl).

Physical data of synthesized compounds given in table 1

Table 1 Physical Data of Synthesized Compounds

\begin{tabular}{|l|l|l|l|l|l|l|l|l|}
\hline S.N & Compound & $\mathbf{R}$ & $\mathbf{R 1}$ & $\mathbf{R 2}$ & M.Pt(C) & Yield(\%) & $\begin{array}{l}\text { Mole. } \\
\text { weight }\end{array}$ & Mole. Formula \\
\hline 1 & $3 \mathrm{a}$ & $-\mathrm{Cl}$ & - & - & $140-145$ & 65 & 257 & $\mathrm{C}_{15} \mathrm{H}_{11} \mathrm{NOCl}$ \\
\hline 2 & $3 \mathrm{~b}$ & $-\mathrm{OH}$ & - & - & $170-172$ & 68 & 272 & $\mathrm{C}_{15} \mathrm{H}_{11} \mathrm{NOCl}_{2}$ \\
\hline 3 & $3 \mathrm{c}$ & - & $-\mathrm{OH}$ & $-\mathrm{OCH} 3$ & $160-165$ & 70 & 261 & $\mathrm{C}_{14} \mathrm{H}_{12} \mathrm{NO}_{2} \mathrm{Cl}$ \\
\hline 4 & $4 \mathrm{a}$ & $-\mathrm{Cl}$ & - & - & $180-185$ & 65 & 347 & $\mathrm{C}_{21} \mathrm{H}_{17} \mathrm{~N}_{3} \mathrm{Cl}$ \\
\hline 5 & $4 \mathrm{~b}$ & $-\mathrm{OH}$ & - & - & $180-185$ & 66 & 364 & $\mathrm{C}_{21} \mathrm{H}_{18} \mathrm{NOCl}$ \\
\hline 6 & $4 \mathrm{c}$ & $-\mathrm{Cl}$ & - & - & $215-220$ & $50-55$ & 458 & $\mathrm{C}_{21} \mathrm{H}_{15} \mathrm{~N}_{5} \mathrm{O}_{4} \mathrm{Cl}_{2}$ \\
\hline 7 & $4 \mathrm{~d}$ & $-\mathrm{OH}$ & - & - & $175-180$ & 55 & 454 & $\mathrm{C}_{21} \mathrm{H}_{16} \mathrm{~N}_{5} \mathrm{O}_{5} \mathrm{Cl}$ \\
\hline 8 & $4 \mathrm{e}$ & - & $-\mathrm{OH}$ & $-\mathrm{OCH}_{3}$ & $185-190$ & $55-60$ & 382 & $\mathrm{C}_{21} \mathrm{H}_{20} \mathrm{~N}_{3} \mathrm{ClO} \mathrm{O}_{2}$ \\
\hline 9 & $4 \mathrm{f}$ & - & $-\mathrm{OH}$ & $-\mathrm{OCH}_{3}$ & $210-215$ & 50 & 483.5 & $\mathrm{C}_{22} \mathrm{H}_{18} \mathrm{~N}_{5} \mathrm{O}_{6} \mathrm{Cl}$ \\
\hline 10 & $5 \mathrm{~d}$ & - & - & - & $170-175$ & 65 & 337.5 & $\mathrm{C}_{19} \mathrm{H}_{16} \mathrm{~N}_{3} \mathrm{ClO}$ \\
\hline 11 & $6 \mathrm{~d}$ & - & - & - & $175-180$ & 57 & 426.5 & $\mathrm{C}_{19} \mathrm{H}_{13} \mathrm{~N}_{5} \mathrm{O}_{5} \mathrm{Cl}$ \\
\hline
\end{tabular}

\section{3-(4-Chlorophenyl)-5-Furan- $N$-Substituted Pyrazolines (5d-6d)}

A mixture of $\mathrm{N}$-(4-chlorophenyl)-3-furan acryl amide (0.01 mole) and 2,4-dinitrophenyl hydrazine/phenyl hydrazine(0.01 mole) in ethanol was refluxed for 5-6 hours. The mixture was concentrated and allows to cool. The resulting solid was filtered, washed, dried and crystallized to get (5d) and (6d).

\section{Characteristics}

$\operatorname{NMR}(\underline{\boldsymbol{\delta}}) \quad$ :-3.35(d,2H,-CH),2.58(s,-NH),7.6(dd,1H,-CH),7.9-8.9(m,Ar-H),11.7(s,1H,Ar-H).

IR $\left(\mathbf{V}_{\max }\right) \mathrm{cm}^{-1} \quad$ :-3286(N-H), 3088(Ar C-H), 1580(C=N), 1615(C=C), 1513(-NO2,), 1220(C-N), 1136 (C-O), 833 (p-sub benzene). 
<smiles>CC(=O)Nc1ccc(Cl)cc1</smiles>

(1)<smiles>[R]c1ccc(C=O)cc1</smiles>

$(2 a-b)$

EtOH $20 \% \mathrm{NaOH}$<smiles>Nc1ccc(Cl)cc1</smiles><smiles>CC(N)=O</smiles>

3a, $\mathrm{R}=-\mathrm{Cl}$

$3 b, \mathrm{R}=-\mathrm{OH}$

(3a-b)<smiles>[R]c1ccc(C2CC(Nc3ccccc3)=NN2c2ccccc2)cc1</smiles>

4a, $\mathrm{R}=-\mathrm{Cl}$

$4 \mathrm{~b}, \mathrm{R}=-\mathrm{OH}$
2,4-Dinitrophenyl hydrazine

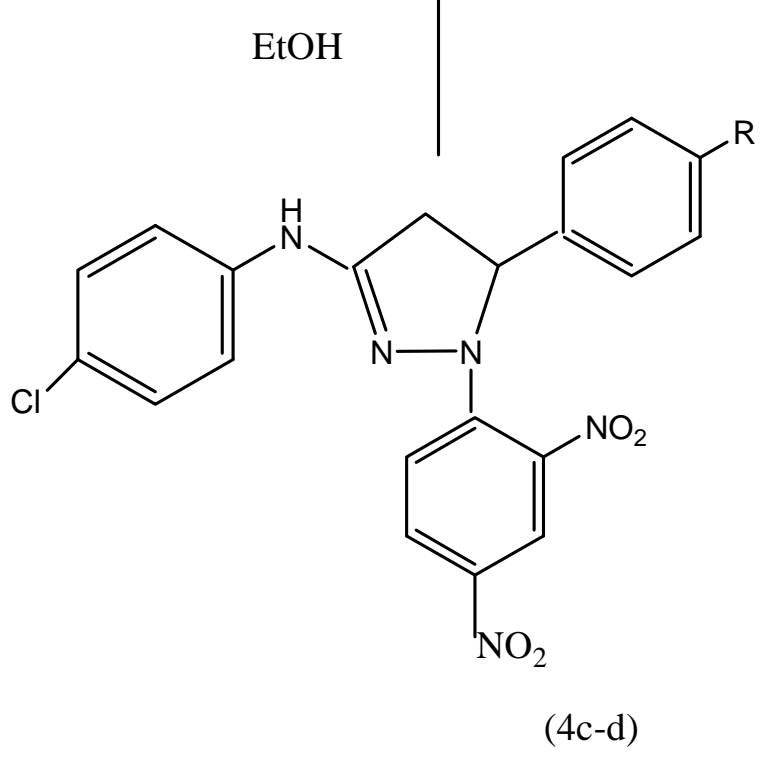

$4 \mathrm{c}, \mathrm{R}=-\mathrm{Cl}$

$4 \mathrm{~d}, \mathrm{R}=-\mathrm{OH}$ 
<smiles>CC(=O)Nc1ccc(Cl)cc1</smiles>

(1)<smiles>[R]c1ccc(C=O)cc1[R]</smiles><smiles>[R2]O[R]O[R]</smiles>

(3c)

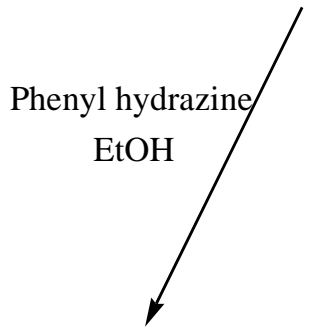<smiles>[R]c1ccc(C2CC(Nc3ccc(Cl)cc3)=NN2c2ccccc2)cc1[R]</smiles>

$\mathrm{R}_{1}=-\mathrm{OH}$

$\mathrm{R}_{2}=-\mathrm{OCH}_{3}$

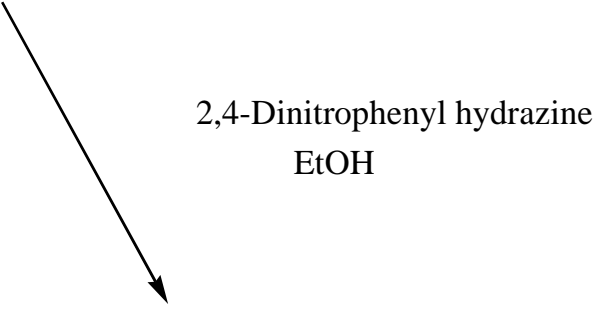<smiles>[R]c1ccc(C2CC(Nc3ccc(Cl)cc3)=NN2c2ccc([N+](=O)[O-])cc2[N+](=O)[O-])cc1[R]</smiles>

(4e) 
Scheme-2<smiles>O=CNc1ccc(Cl)cc1</smiles>

(1)<smiles>O=Cc1ccco1</smiles>

(2d)

$20 \% \mathrm{NaOH}$

$\mathrm{EtOH}$<smiles>O=C(/C=C/c1ccco1)Nc1ccc(Cl)cc1</smiles>

(3d)
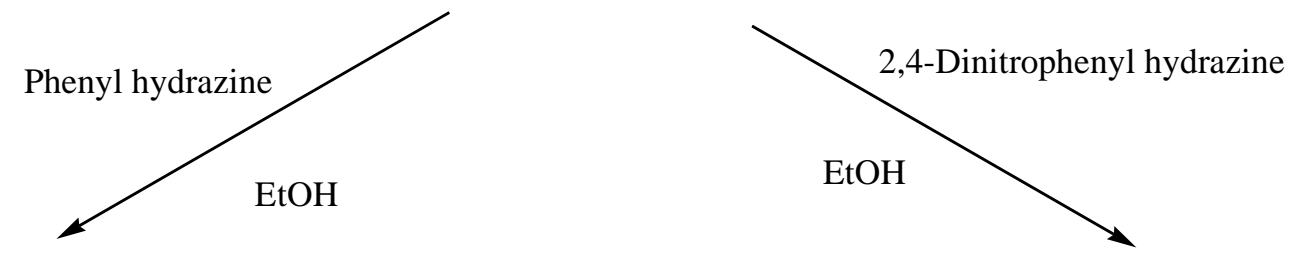<smiles>Clc1ccc(NC2=NN(c3ccccc3)C(c3ccco3)C2)cc1</smiles>

(5d)<smiles>O=[N+]([O-])c1ccc(N2N=C(Nc3ccc(Cl)cc3)CC2c2ccco2)c([N+](=O)[O-])c1</smiles>

$6 d$

\section{Observations}

The spectral studies of synthesized compounds were carried out. The IR spectrum of compound (3c) shows the characteristic band at $1600-1700 \mathrm{~cm}^{-1}$ which confirms the $-\mathrm{C}=\mathrm{O}$ group and band at $1587 \mathrm{~cm}-1$ for $\mathrm{C}=\mathrm{C}$ shows the formation of chalcone. The IR spectra of (4e) shows band at 1500-1600 $\mathrm{cm}-1$ of $\mathrm{C}=\mathrm{N}$ and $\mathrm{H}-\mathrm{NMR}$ spectra shows the dd of $-\mathrm{CH}$ at $7.1 \mathrm{ppm}$ which confirms the formation of pyrazoline ring. The spectral interpretation of (6d) also shows dd of $-\mathrm{CH}$ at $7.6 \mathrm{ppm}$ in $\mathrm{H}-\mathrm{NMR}$ 
spectrum, confirms the formation of pyrazoline and IR spectra shows presence of band at $1580 \mathrm{~cm}-1$ of $\mathrm{C}=\mathrm{N}$ group.

\section{Conclusion}

In conclusion, a simple protocol of synthesis of some novel pyrazoline-3,5 derivatives was developed. From the literature it was revealed that all these synthesized compounds posses good antimicrobial activities.

\section{References}

[1] R.Y. Prasad, L.L. Rao, K .Parsoona, Murli, Ravi Kumar, Bioorg. Chem. Lett.,2005,15,5030.

[2] E.Palaska, D.Erol, R. Deriradmar, Eur.J.Med.Chem,1996,31,43.

[3] O.Zuhal, K.H. Burak, G. Bulent, Eur. J. Med. Chem.,2007,42,403

[4] O. Ahmet, T.J.Gulhan, A.K. Jafer, Eur. J. Med. Chem. 2007,42,403.

[5] G.S. Aysel, Demirayak, C. Gultaze et.al., Eur.J.Med.Chem 2000,35,359.

[6] M.Amir, H. Kumar, S.A. Khan, Bioorg.Med. Chem. Litt.,2008,18,918

[7] Z. Brzozwski, F.S. Czewski, Gdaniec, Euro. J. Med. Chem.,2000,35,1053.

[8] B.f.Abdel-Wahab,H.A.Abdal Aziz,E.M.Ahmed,Eur.J. Med. Chem.

]9] M.I. Husaim, S.Shukla, Indian J.Chem., 1986

[10] V.H.Babu, C.H.Sridevi, A. Joseph, K.K.Shrinivasan,Ind. J. Pharm. Sci. 2007,69,470

[11]F.F. Barsoum, H.M. Hosni, A.S. Girgis, Bioorg.Med.Chem, 2006,14,3929.

[12]Y.K.Agrawal, K.Munna, V.H. Babu, H.Bhatt, P.J.Gogoi, Ind. J. Het.Chem., 2007,16,263.

[13]M.Abid, A. azam, Eur.J.Med.Chem., 2005,40,935. 